\title{
Migration of metallic wire foreign body slicked and instilled in to the lower part of hypopharynx to the neck - a rare case report
}

\begin{abstract}
Ingestion of foreign bodies and migration to extraluminal spaces causing suppurative complications is a rare event. Extraluminal migration of the foreign bodies may cause serious vascular and suppurative complications. We present a rare case of an accidentally ingested foreign body which presented with suppurative complication. The localization of the foreign body was challenging and an open approach was used to remove the foreign body.
\end{abstract}

Keywords: foreign body, migrating foreign body, extraluminal foreign body, metallic foreign body
Volume 10 Issue 3 - 2018

\author{
Trichy Narayanan Janakiram, Udaya \\ Chanukya Samavedam, Kishore Kumar \\ Savaram, Shilpee Bhatia Sharma \\ Royal Pearl Hospital, India
}

\begin{abstract}
Correspondence: Trichy Narayanan Janakiram, Royal Pearl Hospital,Thillai Nagar 3rd cross east, Tiruchirapalli,Tamilnadu, India, Tel+91984246I I76, Email tnjanakiram777@gmail.com
\end{abstract}

Received: October 15, 2016 | Published: May 01, 2018

\section{Case report}

We report the case of a 40 year old female, who presented to our OPD with severe neck pain, fever and swallowing difficulty for the past one week. She had an ingestion of a sharp foreign body eight days back after which she developed pain and difficulty in swallowing. She was advised an X-ray AP and lateral view of neck, which showed a radio-opaque foreign body at the $\mathrm{C} 5$ and $\mathrm{C} 6$ level on the left side (Figure 1). A video laryngoscopic examination was normal. The patient had persistent pain and difficulty in swallowing and developed fever. She was then referred to our centre for further management.

On examination, the patient was conscious and coherent. She was febrile $\left(101^{\circ} \mathrm{F}\right)$, with rest of the vitals stable. The patient had no difficulty in breathing and there was no change of voice. On examination, a diffuse swelling approximately $2.5 \times 2.5 \mathrm{~cm}$ was seen in the left side of the neck, medial to sternocleidomastoid muscle. On palpation over the swelling, it was tender with local temperature raised.

The patient was admitted and the blood investigations were done. The patient was advised an x-ray neck lateral view in which we found a radio-opaque foreign body in the same level, with pre-vertebral soft tissue widening and bamboo stick appearance (straightening of the cervical vertebra) (Figure 2).

The patient underwent a direct laryngoscopy with oesophagoscopic examination under general anesthesia, which did not reveal any abnormality.

The patient was further advised CT scan which revealed a 2.7 $\mathrm{cm}$ linear well defined foreign body with metallic density in the retropharyngeal space at the C5- C6 level (Figure 3). It was localized to be in the left paramedian aspect, superior and posterior to left lobe of thyroid gland and extending posterior to the carotid sheath at that level. A collection of size $3.5 \times 3.2 \mathrm{~cm}$ superior to the left lobe of thyroid gland was seen.

The patient was planned for a neck exploration by an external approach. A vertical incision was given along the anterior border of the sternocleidomastoid from the level of hyoid bone superiorly to the cricoid cartilage inferiorly. The deep fascia was incised along the anterior border of the sternocleidomastoid muscle. The dissection was continued in the paralaryngeal tunnel. Pus collected in the superior pole of the left lobe of thyroid gland pus collected was drained (Figure 2). A sample of pus obtained was sent for culture and sensitivity. On further dissection, the foreign body was seen in horizontal orientation, embedded in the soft tissues medial to the carotid sheath and deep to the superior lobe of the thyroid gland (Figure 3). The foreign body was retrieved and was found to be a metal wire of length $2.7 \mathrm{~cm}$ (Figure 5). Post operative course was uneventful and the patient was discharged after five days.

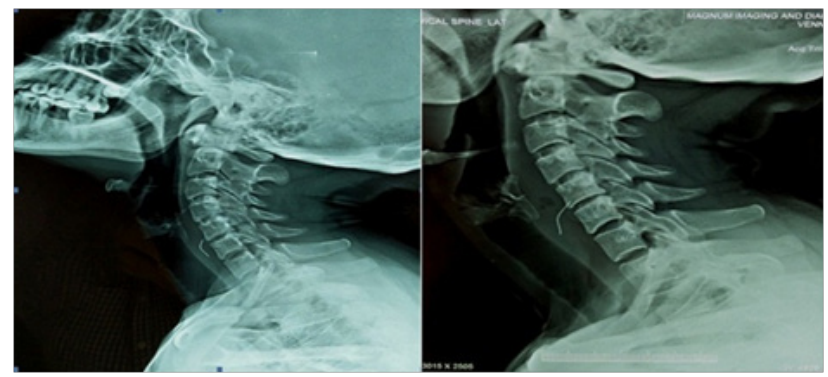

Figure I X-ray showing foreign body (day I and day 8).

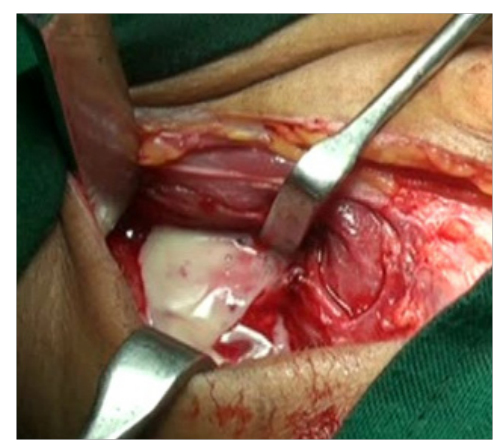

Figure 2 Pus near the superior lobe of the thyroid gland. 


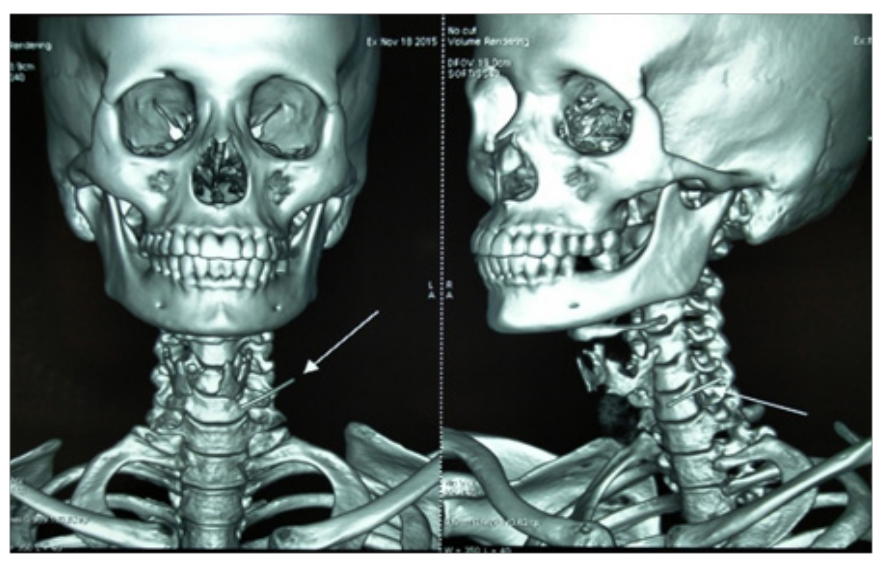

Figure 3 CT scan showing the foreign body.

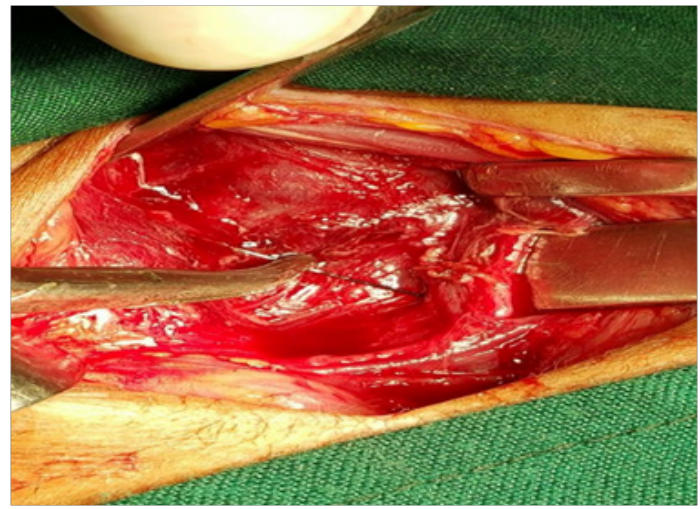

Figure 4 Foreign body seen deep to the superior lobe of the thyroid gland.

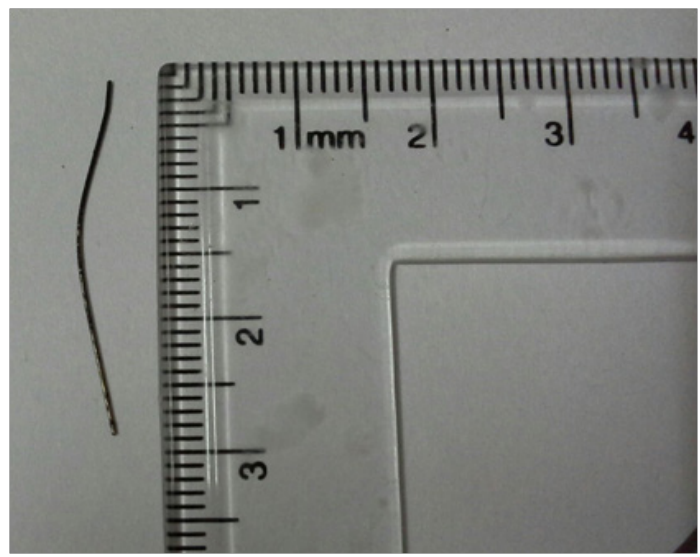

Figure $52.7 \mathrm{~cm}$ metallic foreign body.

\section{Discussion}

The incidence of ingested foreign bodies penetrating the esophagus and going extra luminal into the neck and forming a neck abscess is rare. The perpendicular orientation of the foreign body to the esophageal lumen facilitates the migration. The migrating foreign body paves the way for bacteria to enter the soft tissues of the neck causing suppuration. The incidence of abscess in the neck following extra luminal migration of the foreign body is less than $1 \%{ }^{1,2}$

$\mathrm{X}$ ray, though useful in confirming the presence of foreign body, cannot give a conclusion about the foreign body's precise location. The CT scan is an effective tool that can be used to locate the migrated foreign bodies. ${ }^{3}$ It is an accurate and gives precise location of foreign body compared to a plain X-ray of the neck. The relation of the foreign body to the great vessels of the neck and chest should be studied prior to surgical exploration.

Complications of retained and migrating foreign bodies include esophageal perforation, vascular complications, and cervical abscesses. ${ }^{4-6}$ The dreadful complications include carotid rupture and oesophageal fistula, aortic and subclavian-oesophageal fistula.

Al Muhanna et al.,7 reported a case of foreign body, in which location of any foreign body was unsuccessful even after repeated oesophagoscopy.

A careful clinical and radiological evaluation is necessary to detect extra luminal migration. The present case is unusual, in a way, that patient had a neck abscess following an extra luminal migration of a foreign body, which was localized and surgically managed, thus avoiding life threatening complications. A cervical abscess due to a migrated foreign body can be managed by draining the abscess and retrieving the foreign body.

\section{Conclusion}

Any suspicion of ingestion of a foreign body should not be taken on a lighter note. Absence of foreign body on clinical examination and rigid endoscopy is not confirmatory. This rare case report warrants a high index of suspicion when a foreign body is missed by a rigid esophagoscopy. The persistence of symptoms is an ominous sign of the developing complications. A CT scan of neck is recommended to know the exact size and location of the foreign body, helps in the treatment planning. Immediate exploration of the neck and removal of the foreign body avoids life threatening complications. We report this case because, an ingested foreign body migrated into the neck with cervical abscess is a rare presentation and we managed it successfully.

\section{Consent}

Written informed consent was obtained from the patient for publication of this case report and any accompanying images. A copy of the written consent is available for review by the editor in chief of this journal.

\section{Acknowledgments \\ None.}

\section{Competing interests}

We declare that there are no competing interests in submitting this article.

\section{References}

1. Lam HC, Woo JK, van Hasselt CA. Esophageal perforation and neck abscess from ingested foreign bodies: Treatment and outcomes. Ear Nose Throat J. 2003;82(10):786-794.

2. Nandi P, Ong GB. Foreign body in the oesophagus: Review of 2394 cases. Br J Surg. 1978;65(1):5-9.

3. Braverman I, Gomori JM, Polv O, et al. The role of CT imaging in the evaluation of cervical esophageal foreign bodies. $J$ Otolaryngol. 1993;22(4):311-314. 
4. Watanabe K, Amano M, Nakanome A, et al. The prolonged presence of a fish bone in the neck. Tohoku J Exp Med. 2012;227(1):49-52.

5. Landis BM, Giger R. An unusual foreign body migrating through time and tissues. Head Face Med. 2006; 2:30.
6. McLaughlin RT, Morris JD, Haight C. The morbid nature of migrating foreign body in the esophagus. J Thorac Cardiovasc Surg. 1968; 55(2):188-192.

7. Muhanna AA, Abu Chra KA, Dasti H, et al. Thyroid lobectomy for removal of a fish bone. J Laryngol Otol. 1990;104(6):511-512. 